PACIFIC JOURNAL OF MATHEMATICS

Vol. 186, No. 1, 1998

\title{
A NOTE ON TAYLOR'S BRAUER GROUP
}

\author{
S. Caenepeel and F. Grandjean
}

We make clear that some of the properties of central separable algebras without unit, as announced by Taylor and Raeburn, are only valid under the assumption that the algebra in question is flat. This leads to the introduction of two possibly different versions of the big Brauer group. Both of them inject into the second étale cohomology group, and one of them is isomorphic to it.

Let $R$ be a commutative ring. Taylor introduced a Brauer group consisting of classes of algebras that do not necessarily have a unit. The classical Brauer group $\operatorname{Br}(R)$ is contained in this new Brauer group, and one of the exciting properties is that the new Brauer group is isomorphic to the second étale cohomology group $H^{2}\left(R_{e ́ t}, \mathbb{G}_{m}\right)$. One of the classical results in the theory of the Brauer group is Gabber's Theorem stating that the Brauer group $\operatorname{Br}(R)$ is isomorphic to the torsion part of $H^{2}\left(R_{e ́ t}, \mathbb{G}_{m}\right)$. Thus Taylor's Brauer group provides an algebraic description of the non-torsion elements in $H^{2}\left(R_{e ́ t}, \mathbb{G}_{m}\right)$.

The idea is that the algebras that represent elements of the big Brauer group are central separable algebras in a generalized sense. These central separable algebras are in general not projective or finitely generated. Here a logical problem arises, comparable to the problems that one has if one tries to introduce the Grothendieck group of all projective modules: one has to make sure that the equivalence classes of central separable algebras form a set. To overcome this problem, Taylor proved that every central separable algebra $A$ is equivalent to a subalgebra that is contained in a finitely generated $R$ module. Unfortunately, we have discovered that this property is not true in general. We will explain that its proof relies on another general property that is only true if the central separable algebra $A$ in question is flat as an $R$-module. We have a counterexample in the situation where $A$ is not flat.

We have investigated that the flatness of $A$ is also used in several other properties that were proved by Taylor and Raeburn. Apart from the logical problem, there is also the fact that the splitting Theorem, stating that every central separable algebra is a twisted form of a so-called elementary algebra, no longer holds. The importance of this property is illustrated by the fact that it is the basis for the construction of the monomorphism from the Brauer group to the second cohomology group. 
In this note, we discuss the properties of central separable algebras that require flatness. We propose two modified definitions of the big Brauer group. $\mathrm{BR}(R)$ consists of equivalence classes represented by a flat central separable algebra, while $\operatorname{Br}^{\prime}(R)$ consists of equivalence classes of finitely generated central separable algebras. It will turn out that $\mathrm{BR}(R) \subset \mathrm{Br}^{\prime}(R)$. We do not know if this inclusion is an equality. Furthermore, both Brauer groups map injectively into the second étale cohomology group, and we can show that $\operatorname{Br}^{\prime}(R)$ is isomorphic to $H^{2}\left(R_{e ́ t}, \mathbb{G}_{m}\right)$. Thus one of the principal properties of the big Brauer group is saved.

We end this introduction with some historical remarks. In the classical situation where one considers only algebras with a unit, central separable algebras have been renamed as Azumaya algebras, and this is the terminology that is mostly used nowadays. There is also a difference in approach: in the original paper by Auslander and Goldman [2], the algebras in question have to be central and separable. Another, more conceptual, approach is possible: given an algebra $A$, one has a pair of adjoint functors between the categories of $R$-modules and $A$-bimodules (see for example [9]). $A$ is an Azumaya algebra if and only if these adjoint functors establish a category equivalence. In a forthcoming monograph [3], the first author develops the corresponding theory of Azumaya algebras without a unit.

\section{Regular modules over flat central separable algebras.}

Let $R$ be a commutative ring with unit, and $A$ an associative $R$-algebra, not necessarily with a unit. The center $Z(A)$ of $A$ is by definition $\operatorname{End}_{A^{\mathrm{e}}}(A)$. $A$ is called $R$-central if the canonical map $R \rightarrow Z(A)$ is an isomorphism. $A$ is called $R$-separable if the following conditions are satisfied.

(1) $A^{2}=A$

(2) $A$ is a projective $A^{\mathrm{e}}$-module

(3) $m A \neq A$ for any maximal ideal $m$ of $Z(A)$.

If $A$ has a unit, then $A$ is separable if and only if $A$ is separable in the sense of [4], and $A$ is central separable if and only if $A$ is central separable (or Azumaya) in the classical sense. We refer to [2], [4], [9] or [11] for an introduction to the theory of Azumaya algebras.

The basic examples of central separable algebras are the so-called elementary algebras (cf. [14]). We recall that they can be obtained in the following way: Consider two $R$-modules $P$ and $P^{\prime}$, and a surjective map $\lambda: P^{\prime} \otimes P \rightarrow R$. The elementary algebra $E_{R}\left(P, P^{\prime}, \lambda\right)$ associated to $P, P^{\prime}$ and $\lambda$ is the $R$-module $P \otimes P^{\prime}$ together with the multiplication given by the 
formula

$$
\left(p \otimes p^{\prime}\right)\left(q \otimes q^{\prime}\right)=\lambda\left(p^{\prime} \otimes q\right) p \otimes q^{\prime} .
$$

According to $\left[\mathbf{1 4}\right.$, Prop. 4.5], the elementary algebra $E_{R}\left(P, P^{\prime}, \lambda\right)$ has a unit if and only if $P$ and $P^{\prime}$ are faithfully projective as $R$-modules, $P^{\prime} \cong P^{*}$ and $\lambda$ is the duality map $P^{*} \otimes P \rightarrow R$. In this situation, $E_{R}\left(P, P^{\prime}, \lambda\right)$ is nothing else but the endomorphism ring of $P$. In the theory of the big Brauer group, the elementary algebras play the role that endomorphism rings play in the classical theory.

Now let $A$ be an $R$-algebra, and $M$ a (left) $A$-module. Recall from [14] that $M$ is called $A$-regular if the map $A \otimes_{A} M \rightarrow M$ induced by the scalar multiplication on $M$ is an isomorphism. $A$ is called a regular algebra if it is regular over itself.

Proposition 1.1. Let $A$ be an $R$-algebra and $M$ a (left) $A$-module. If the scalar multiplication $\mu: A \otimes_{R} M \rightarrow M$ has a A-linear section, then $M$ is regular. Furthermore, if $A$ is separable, then the converse property also holds.

Proof. Having a section, the map $\mu$ is surjective, so we only have to show that $A \otimes_{A} M \rightarrow M$ is injective. Consider $x=\sum_{i} a_{i} \otimes m_{i} \in A \otimes_{R} M$, and assume that $\mu(x)=\sum_{i} a_{i} m_{i}=0$. Write $s\left(m_{i}\right)=\sum_{j} a_{i j} \otimes m_{i j}$. Then $\sum_{i, j} a_{i} a_{i j} \otimes m_{i j}=s\left(\sum_{i} a_{i} m_{i}\right)=0$, and $x=\sum_{i, j} a_{i} \otimes a_{i j} m_{i j}-\sum_{i, j} a_{i} a_{i j} \otimes m_{i j}=$ 0 in $A \otimes_{A} M$.

If $A$ is separable, then conditions (1) and (2) of the definition of separability imply that the multiplication map $A \otimes_{R} A \rightarrow A$ has an $A^{\mathrm{e}}$-linear section $\varphi$. For a regular $A$-module $M$, we construct a section of the map $\mu$ as the following composition:

$$
M \cong A \otimes_{A} M \stackrel{\varphi \otimes M}{\longrightarrow} A \otimes_{R} A \otimes_{A} M \cong A \otimes_{R} M .
$$

We conclude from this result that a separable $R$-algebra is always regular. Now let $A$ be a separable $R$-algebra, and assume that $M$ is a regular left $A$-module. In [14, Prop. 1.6], it is shown that an $A$-submodule of $N$ with surjective scalar multiplication is regular. The following counterexample shows that this result is not allways true.

Example 1.2. Let $A=E_{\mathbb{Z}}(\mathbb{Z}, \mathbb{Z} / 2 \mathbb{Z} \oplus \mathbb{Z}, \lambda)$, where $\lambda$ is defined by the formula

$$
\lambda((\bar{z}, t) \otimes s)=t s
$$


As a $\mathbb{Z}$-module, $A \cong \mathbb{Z} / 2 \mathbb{Z} \oplus \mathbb{Z}$, with multiplication given by

$$
\left(\bar{z}_{1}, t_{1}\right) \cdot\left(\bar{z}_{2}, t_{2}\right)=\left(t_{1} \bar{z}_{2}, t_{1} t_{2}\right) .
$$

Take $a=(\overline{0}, 2) \in A$. Then the principal ideal $a A \cong 2 \mathbb{Z}$ is not regular as an $A$-module. Indeed the image of $(\overline{0}, 2) \otimes(\overline{1}, 0)$ in $a A \otimes_{A} A$ under the multiplication map is zero, while $(\overline{0}, 2) \otimes(\overline{1}, 0) \neq 0$. To see this, consider the well defined homomorphism $a A \otimes_{A} A \rightarrow \mathbb{Z} / 2 \mathbb{Z}$ sending $(\overline{0}, 2 s) \otimes(\bar{z}, t)$ to $s \bar{z}$. This morphism sends $(\overline{0}, 2) \otimes(\overline{1}, 0)$ to $\overline{1}$ which is not zero in $\mathbb{Z} / 2 \mathbb{Z}$.

The elementary algebra $A$ considered in Example 1.2 is not flat as a $\mathbb{Z}$ module, and this is why we have problems. In the proof of [14, Prop. 1.6], the flatness of $A$ as an $R$-module is implicitly used. The correct statement is the following:

Proposition 1.3. Let $A$ a separable, flat $R$-algebra and $M$ a regular (left) $A$-module. If $N$ is an $A$-submodule of $M$ such that $N=A N$, then $N$ is regular as an $A$-module.

Proof. If we can show that $A$ is flat as an $A$-module, then the regularity of $N$ follows immediately after we inspect the following commutative diagram:

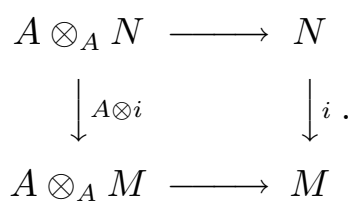

Here $i: N \rightarrow M$ is the inclusion.

Let us show that $A$ is flat as an $A$-module. $A \otimes_{A} M$ fits into an exact sequence

$$
A \otimes_{R} A \otimes_{R} M \stackrel{\lambda}{\rightarrow} A \otimes_{R} M \stackrel{\pi}{\rightarrow} A \otimes_{A} M \rightarrow 0
$$

where $\lambda$ is defined by $\lambda(a \otimes b \otimes m)=a b \otimes m-a \otimes b m$, and $\pi$ is the canonical surjection. Let $\varphi: A \rightarrow A^{\mathrm{e}}$ be an $A^{\mathrm{e}}$-linear section of the multiplication map ( $A$ is separable). It is easy to see that

$$
\lambda \circ(\varphi \otimes M): A \otimes_{R} M \rightarrow \operatorname{Im}(\lambda)
$$

is a projection. Therefore the exact sequence of $A$-modules

$$
0 \rightarrow \operatorname{Im}(\lambda) \rightarrow A \otimes_{R} M \stackrel{\pi}{\rightarrow} A \otimes_{A} M \rightarrow 0
$$

is split, and $\pi$ has an $A$-linear section $s: A \otimes_{A} M \rightarrow A \otimes_{R} M$. Now consider an exact sequence of $A$-modules

$$
0 \rightarrow N \stackrel{f}{\rightarrow} M \stackrel{g}{\rightarrow} L \rightarrow 0
$$


and look at the commutative diagram

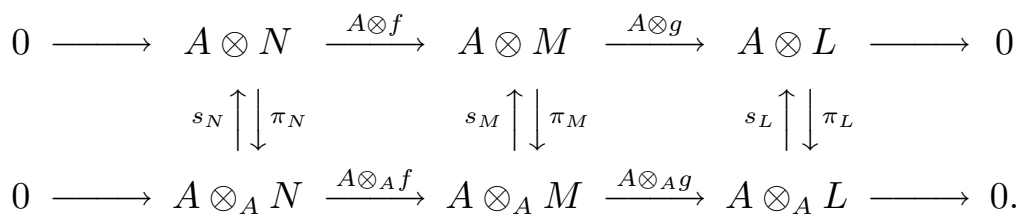

The top row is exact since $A$ is flat as an $R$-module, and this implies easily that the bottom row is also exact. It follows that $A$ is flat as an $A$ module.

\section{Morita equivalence and the big Brauer group.}

Two regular algebras $A$ and $B$ are called Morita equivalent if there exist a regular $A$ - $B$-bimodule $M$ and a regular $B$ - $A$-bimodule $N$ such that we have two bimodule isomorphisms $f: M \otimes_{B} N \stackrel{\cong}{\rightrightarrows} A$ and $g: N \otimes_{A} M \stackrel{\cong}{\rightrightarrows} B$ that are associative in the following sense: $f(m \otimes n) \cdot m^{\prime}=m \cdot g\left(n \otimes m^{\prime}\right)$ and $g(n \otimes m) \cdot n^{\prime}=n \cdot f\left(m \otimes n^{\prime}\right)$ for all $m, m^{\prime} \in M, n, n^{\prime} \in N$. An easy modification of the classical Morita theorem shows that the functors $M \otimes_{B} \bullet$ and $N \otimes_{A} \bullet$ establish an equivalence between the categories of regular left $A$-modules and regular left $B$-modules if $A$ and $B$ are Morita equivalent. It is also straightforward to show that Morita equivalence is an equivalence relation.

Also recall the following generalization of elementary algebras. Let $A$ be a regular $R$-algebra, $M$ a right regular $A$-module, $N$ a left regular $B$-module, and $\lambda: \quad N \otimes_{R} M \rightarrow A$ a surjective $A^{\text {e}}$-linear map. The $A$-elementary algebra $E_{A}(M, N, \lambda)$ associated to $M, N$ and $\lambda$ is the $R$-module $M \otimes_{A} N$, with multiplication given by the formula

$$
(m \otimes n)\left(m^{\prime} \otimes n^{\prime}\right)=m \cdot \lambda\left(n \otimes m^{\prime}\right) \otimes n^{\prime} .
$$

In [14, Prop. 4.2], the following result is stated: If $A$ is a central separable algebra, and $B$ is an $R$-algebra, then $A$ and $B$ are Morita equivalent if and only if $B$ is an $A$-elementary algebra. The proof uses the fact that $A$ is flat as an $R$-module, so one expects that the result is true only in the case where $A$ is flat. We were able to find a proof that avoids the flatness of $A$.

First we recall a result from category theory. Let $f, g: M \rightarrow N$ be two morphisms in $R$-mod, where $R$ is an arbitrary ring. The arrow $q: N \rightarrow P$ is called the absolute coequalizer of $f$ and $g$ if $q \circ f=q \circ g, q$ has a section $r, g$ has a section $s$, and

$$
f \circ s=r \circ q .
$$


An absolute coequalizer is always a coequalizer: Take $n \in N$, and assume that $q(n)=0$. Then

$$
g(s(n))-f(s(n))=n-r(q(n))=n .
$$

Proposition 2.1. Let $A$ and $B$ be $R$-algebras, and suppose that $A$ is separable. Then $B$ is Morita equivalent to $A$ if and only if $B$ is A-elementary.

Proof. One implication is obvious, we refer to [14]. Conversely, suppose that $B=E_{A}(M, N, \lambda)$ is an $A$-elementary algebra. Then $M$ is a left $B$-module, the left $B$-action is given by the formula

$$
(m \otimes n) \cdot m^{\prime}=m \cdot \lambda\left(n \otimes m^{\prime}\right) .
$$

In a similar way, $N$ is a right $B$-module.

We know that the multiplication map on $A$ has an $A^{\mathrm{e}}$-linear section $\varphi$ : $A \rightarrow A^{\mathrm{e}}$. We will use the following Sweedler-type notation: For $a \in A$, we write

$$
\varphi(a)=\sum a^{1} \otimes a^{2}
$$

From Proposition 1.1, we know that the scalar multiplication $M \otimes_{R} A \rightarrow M$ has a section $t: M \rightarrow M \otimes_{R} A$ given by the formula

$$
t(m \cdot a)=\sum m \cdot a^{1} \otimes a^{2}
$$

for all $m \in M$ and $a \in A$. $A$ is $A^{\mathrm{e}}$-projective, so the surjective map $\lambda$ : $N \otimes_{R} M \rightarrow A$ has an $A^{\mathrm{e}}$-linear section $s$. We will write

$$
s(a)=\sum a^{N} \otimes a^{M} \in N \otimes M
$$

for all $a \in A$. We claim that the scalar multiplication map $B \otimes_{R} M \rightarrow M$ is split. The splitting map $s$ is the composition

$$
s=(\pi \otimes M) \circ(M \otimes r) \circ t
$$

where $\pi: M \otimes_{R} N \rightarrow M \otimes_{A} N$ is the canonical projection. In other words,

$$
s(m \cdot a)=\sum\left(m a^{1} \otimes\left(a^{2}\right)^{N}\right) \otimes\left(a^{2}\right)^{M} .
$$

Now

$\sum\left(m a^{1} \otimes\left(a^{2}\right)^{N}\right) \cdot\left(a^{2}\right)^{M}=\sum m a^{1} \cdot \lambda\left(\left(a^{2}\right)^{N} \otimes\left(a^{2}\right)^{M}\right)=\sum m \cdot a^{1} a^{2}=m a$

as needed. 
We now claim that $\lambda: N \otimes_{R} M \rightarrow A$ is the absolute coequalizer of

$$
\left\{\begin{array}{l}
\psi_{N}^{r} \otimes M \\
N \otimes \psi_{M}^{l}
\end{array} \quad: N \otimes_{R} B \otimes_{R} M \longrightarrow N \otimes_{R} M .\right.
$$

Indeed, $\lambda \circ\left(\psi_{N}^{r} \otimes M\right)=\lambda \circ\left(N \otimes \psi_{M}^{l}\right), r$ is a section of $\lambda$ and $N \otimes s$ is a section of $N \otimes \psi_{M}^{l}$. Finally

$$
\left(\psi_{N}^{r} \otimes M\right) \circ(N \otimes s)=r \circ \lambda
$$

since

$$
\begin{aligned}
& \left(\psi_{N}^{r} \otimes M\right)((N \otimes s)(n \otimes m \cdot a)) \\
& =\left(\psi_{N}^{r} \otimes M\right)\left(\sum n \otimes\left(m a^{1} \otimes\left(a^{2}\right)^{N}\right) \otimes\left(a^{2}\right)^{M}\right) \\
& =\sum \lambda\left(n \otimes m \cdot a^{1}\right) \cdot\left(a^{2}\right)^{N} \otimes\left(a^{2}\right)^{M} \\
& =\sum \lambda\left(n \otimes m \cdot a^{1}\right) r\left(a^{2}\right) \\
& =\sum r\left(\lambda\left(n \otimes m \cdot a^{1}\right) \cdot a^{2}\right) \\
& =\sum r\left(\lambda\left(n \otimes m \cdot a_{1} \cdot a^{2}\right)\right)=(r \otimes \lambda)(n \otimes m \cdot a)
\end{aligned}
$$

for all $m \in M, n \in N$ and $a \in A . N \otimes_{B} M$ and $A$ are both coequalizers of (1), so it follows from the universal property of coequalizers that the map $\bar{\lambda}: N \otimes_{B} M \rightarrow A$ induced by $\lambda$ is an isomorphism. $\left(B, A, M, N, I_{B}, \bar{\lambda}\right)$ is a strict Morita context.

We have saved Proposition 4.2 of [14]. An important consequence is that Proposition 4.3, which follows from Proposition 4.2, is also true in general. We mention it explicitly, as we will need it further on.

Proposition 2.2. Let $N$ be a regular left ideal and $M$ a regular right ideal of the central separable algebra $A$. If $N M=A$, then the subalgebra $M N$ is central separable and Morita equivalent to $A$.

In [14], Taylor introduces the big Brauer group of central separable algebras as the set of Morita equivalence classes of central separable algebras. Here we face the following logical problem: how do we know that Morita equivalence classes form a set? The explanation given by Taylor is the following: he shows that every central separable $R$-algebra $A$ is Morita equivalent to a subalgebra which is contained in a finitely generated $R$-submodule of $A$ (cf. [14, Corollary 4.1]). From this result, it follows indeed that equivalence classes of central separable algebras form a set. 
Unfortunately, the proof of [14, Corollary 4.1] relies on [14, Proposition 1.6], and we have seen that this property fails to be true if $A$ is not flat as an $R$-module. In the proof of [14, Corollary 4.1], we need the fact that ideals of $A$ of the form $\sum_{i} a_{i} A$ are regular. For a correct statement, we therefore need the additional assumption that $A$ is flat as an $R$-module.

Proposition 2.3. Every central separable $R$-algebra $A$ which is flat as $R$ module, is Morita equivalent to a subalgebra which is contained in a finitely generated $R$-submodule of $A$. In particular, if $R$ is noetherian then every central separable flat $R$-algebra is Morita equivalent to a subalgebra which is a finitely generated as an $R$-module.

Remark that it does not follow from the proof of Proposition 2.3 that the finitely generated subalgebra is itself flat as an $R$-module.

We conclude that we are no longer sure whether the equivalence classes of central separable algebras form a set. We present two ways to escape this problem.

The first one is to restrict attention to equivalence classes that are represented by flat central separable algebras. Using Proposition 2.3, we see that these classes form a set, and the operation induced by the tensor product puts an abelian group structure on this set. Indeed, the neutral element is represented by $R$ itself, which is obviously flat as an $R$-module, and, if $A$ is a flat central separable $R$-algebra, then then the inverse of $[A]$ is represented by $A^{\text {op }}$, which is also flat as an $R$-module. The Brauer group that we obtain is denoted by $\mathrm{BR}(R)$.

We can also look at Morita equivalence classes that are represented by a finitely generated central separable algebra. Then we obviously obtain a set, and the tensor product makes this set into a group which we will denote by $\operatorname{Br}^{\prime}(R)$. This notation is inspired by the notation $\mathrm{K}_{0}^{\prime}(R)$ for the Grothendieck group of the category of finitely generated $R$-modules. It is obvious that $\mathrm{BR}(R)$ and $\operatorname{Br}^{\prime}(R)$ both contain the classical Brauer group $\operatorname{Br}(R)$ as a subgroup. If $R$ is noetherian, then it follows from Proposition 2.3 that $\mathrm{BR}(R) \subset \mathrm{Br}^{\prime}(R)$. We will see below that this inclusion holds also in the case where $R$ is not noetherian. We do not know whether $\operatorname{BR}(R)=\operatorname{Br}^{\prime}(R)$.

\section{Cohomological interpretation of $\mathrm{BR}(R)$ and $\operatorname{Br}^{\prime}(R)$.}

It is well-known that the classical Brauer group $\operatorname{Br}(R)$ may be embedded into the second étale cohomology group $H^{2}\left(R_{e ́ t}, \mathbb{G}_{m}\right)$, see e.g. [7], [9], [10]. This embedding is based on the splitting Theorem, stating that every Azumaya algebra is a twisted form of a matrix ring. A similar result for central separable algebras is stated in [12, Lemma 2.1]. The proof uses [14, Corollary 4.1], 
and we have seen above that this result is uncertain if $A$ is not flat. We can repair the proof, either by assuming that $A$ is flat, and applying Proposition 2.3 , or by assuming that $A$ is finitely generated as an $R$-module, in which case we do not need Proposition 2.3. We then obtain the following result. Recall from [14] that an idempotent $e$ of $A$ is called a rank one idempotent if $e A e=R e \cong R$. If $A$ contains a rank one idempotent $e$, then $A$ is Morita equivalent to $R$, and $A$ is an $R$-elementary algebra. Indeed, observe that $e A$ and $A e$ are respectively a right and a left regular $A$-module (use the fact that $e$ is an idempotent), and apply Proposition 2.2 with $M=e A$ and $N=A e$. It follows that $e A^{2} e=e A e=R e \cong R$ is Morita equivalent to $A$. The splitting theorem can now be stated as follows.

Theorem 3.1. Let $A$ be an $R$-algebra that is flat or finitely generated as an $R$-module. Then the following assertions are equivalent:

(1) $A$ is a central separable $R$-algebra;

(2) $A \otimes_{R} S$ is a central separable $S$-algebra containing a rank one idempotent for some étale covering $S$ of $R$;

(3) $A \otimes_{R} S$ is an $S$-elementary algebra for some étale covering $S$ of $R$;

(4) $A \otimes_{R} S$ is a central separable $S$-algebra containing a rank one idempotent for some commutative faithfully flat $R$-algebra $S$;

(5) $A \otimes_{R} S$ is an $S$-elementary algebra for some commutative faithfully flat $R$-algebra $S$;

(6) $A \otimes_{R} S$ is a central separable $S$-algebra containing a rank one idempotent for some commutative faithfully flat $R$-algebra $S$ that is locally of finite type;

(7) $A \otimes_{R} S$ is an $S$-elementary algebra for some commutative faithfully flat $R$-algebra $S$ that is locally of finite type.

The set $\Omega$ of all $A^{\mathrm{e}}$-bimodule morphisms $A_{l} \otimes A_{r} \rightarrow A^{\mathrm{e}}$ is called the Goldman set. If $A$ is central separable, then it is not difficult to show that $\Omega$ is projective of rank one as an $R$-module, and that it is of order at most 2 in the Brauer group (see [14, Proposition 3.7]). From the splitting theorem, one can deduce that there exists an $A^{\mathrm{e}}$-bimodule isomorphism $\omega: A_{l} \otimes A_{r} \rightarrow A^{\mathrm{e}}$, and we have the following result.

Corollary 3.2. If $A$ is a central separable algebra that is flat or finitely generated as an $R$-module, then the Goldman set is a free module of rank one.

In [12], this result is stated for arbitrary central separable algebras. It is an open question whether the Goldman set is free of rank one for an arbitrary central separable algebra. 
Theorem 3.3. $\mathrm{BR}(R)$ and $\operatorname{Br}^{\prime}(R)$ embed into $H^{2}\left(R_{\text {ét }}, \mathbb{G}_{m}\right)$.

Proof. The proof is relatively simple if one uses Artin's Refinement Theorem [1]. A consequence of Artin's Theorem is that the étale cohomology groups can be written as inductive limits of Amitsur cohomology groups:

$$
H^{2}\left(R_{e ́ t}, \mathbb{G}_{m}\right)=\underline{\lim } H^{2}\left(S / R, \mathbb{G}_{m}\right)
$$

where the inductive limit runs over all étale coverings $S$ of $R$. Take a central separable algebra $[A]$ that is flat or finitely generated as an $R$-module. From Theorem 3.1, we know that $A \otimes_{R} S$ is $S$-elementary for some étale covering $S$ of $R$. Thus we have an isomorphism $\sigma: A \otimes S \rightarrow E_{S}\left(P, P^{\prime}, \lambda\right)=E_{S}(\underline{P})$. We define $\Phi$ by the commutativity of the following diagram.

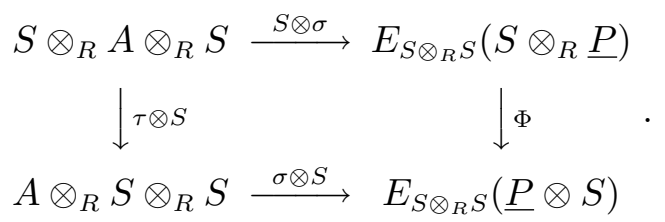

From the fact that $S \otimes_{R} S$ and $E_{S \otimes_{R} S}\left(S \otimes_{R} \underline{P}\right)$ are Morita equivalent, one can deduce that $\Phi$ is of the following type.

$$
\begin{aligned}
& \Phi:\left(S \otimes_{R} P\right) \otimes_{S \otimes_{R} S}\left(S \otimes P^{\prime}\right) \\
& \cong\left(S \otimes_{R} P\right) \otimes_{S \otimes_{R} S} I \otimes_{S \otimes_{R} S} I^{*} \otimes_{S \otimes_{R} S}\left(S \otimes P^{\prime}\right) \\
& \stackrel{f \otimes f^{\prime}}{\longrightarrow}\left(P \otimes_{R} S\right) \otimes_{S \otimes_{R} S}\left(P^{\prime} \otimes S\right)
\end{aligned}
$$

for some invertible $S \otimes_{R} S$-module $I$. Using Artin's Refinement Theorem a second time, we see that we can replace $S$ by a étale covering of $S$ in such a way that $I$ is free of rank one as an $S \otimes_{R} S$-module. Then we obtain an isomorphism $f: S \otimes_{R} P \rightarrow P \otimes_{R} S$, and an easy argument shows that $f_{2}^{-1} \circ f_{3} \circ f_{1}: S \otimes_{R} S \otimes_{R} P \rightarrow S \otimes_{R} S \otimes_{R} P$ is given by multiplication by a unit $u$ in $S^{\otimes 3}$ which is an Amitsur cocycle. Sending [A] to [u], we obtain welldefined monomorphisms from $\operatorname{BR}(R)$ and $\operatorname{Br}^{\prime}(R)$ into $H^{2}\left(R_{e ́ t}, \mathbb{G}_{m}\right)$. This can be verified along the lines of the corresponding proof for the classical Brauer group $\operatorname{Br}(R)$, as exhibited in [9, Sec. 5.2].

Our Theorem can also be proved without using Artin's Refinement Theorem. For full detail, we refer to the forthcoming [3].

Recall that a commutative $R$-algebra $S$ (not necessarily having a unit) is called quasi-finite if $S \otimes k(p)$ is finite dimensional as a $k(p)$-vector space, for every prime ideal $p$ of $R$. A map $\tau: S \rightarrow R$ is called a trace map if $\tau \otimes k(p): S \otimes k(p) \rightarrow k(p)$ coincides with the standard trace map for every prime ideal $p$ of $R$. 
Now let $f: R \rightarrow S$ be a noetherian commutative $R$-algebra, with $R$ and $S$ noetherian. We say that $S$ is of trace type if we have a factorization

$$
R \stackrel{g}{\rightarrow} T \stackrel{i}{\rightarrow} S
$$

with $T$ finitely generated as an $R$-module, $i: T \rightarrow S$ an open immersion, and such that there exists an ideal $I$ of $T$ with $D(I)=\operatorname{Spec}(S)$ and $I$ having a trace map.

A key result is Taylor's Refinement Theorem ([12, Lemma 3.4]): If $S$ is a an étale covering of $R$, then there exists an étale covering $S \rightarrow S^{\prime}$ such that $R \rightarrow S^{\prime}$ is of trace type. The proof of this result is based on Zariski's Main Theorem and the Artin-Rees Lemma. It can be used to prove the next Theorem.

\section{Theorem 3.4.}

$$
\operatorname{Br}^{\prime}(R) \cong H^{2}\left(R_{e ́ t}, \mathbb{G}_{m}\right) .
$$

Proof. Assume first that $R$ is noetherian. Let $c \in H^{2}\left(R_{e ́ t}, \mathbb{G}_{m}\right)$. Using Artin's Refinement Theorem, we can assume that $u$ is represented by a cocycle $u \in Z^{2}\left(S / R, \mathbb{G}_{m}\right)$ for some étale covering $S$ of $R$. Invoking Taylor's Refinement Theorem, we can assume that $S$ is of trace type. Let $g, i, T$ and $I$ be as above, and consider

$$
\left\{\begin{array}{l}
p_{1} \\
p_{2}
\end{array} \quad: T \otimes S \Longrightarrow T \otimes S \otimes S: t \otimes s \mapsto\left\{\begin{array}{l}
t \otimes 1 \otimes s \\
t \otimes s \otimes 1
\end{array} .\right.\right.
$$

Let $S \otimes \underline{S} \otimes S$ be $S^{\otimes 3}$ considered as a $T \otimes S$-module, with $T$ and $S$ acting respectively on the first and third factor. Then $I \otimes \underline{S} \otimes S$ is a $T \otimes S$-submodule of $S \otimes \underline{S} \otimes S$, and we can consider the $T \otimes S$-modules

$$
\begin{aligned}
M & =(I \otimes \underline{R} \otimes S) \cap u^{-1}(I \otimes \underline{S} \otimes S) \\
N & =(I \otimes \underline{R} \otimes S) \cap u(I \otimes \underline{S} \otimes S)
\end{aligned}
$$

and let

$$
\begin{aligned}
M_{i} & =M \otimes_{T \otimes S, p_{i}}(T \otimes S \otimes S) \\
N_{i} & =N \otimes_{T \otimes S, p_{i}}(T \otimes S \otimes S)
\end{aligned}
$$

for $i=1,2$. With this notation, we mean that $T \otimes S \otimes S$ is considered as a $T \otimes S$-module via $p_{i} . M_{1}$ and $M_{2}$ are $T \otimes S \otimes S$-submodules of $S \otimes S \otimes S$, and we claim that

$$
u_{2} M_{1}=M_{2} \text { and } u_{2} N_{2}=N_{1} \text {. }
$$


Following Sweedler's tradition, we write

$$
u=\sum u^{1} \otimes u^{2} \otimes u^{3} \in S^{\otimes 3} \text { and } u^{-1}=\sum v^{1} \otimes v^{2} \otimes v^{3} \in S^{\otimes 3} .
$$

Also recall the following notations

$$
u_{1}=\sum 1 \otimes u^{1} \otimes u^{2} \otimes u^{3}, \quad u_{2}=\sum u^{1} \otimes 1 \otimes u^{2} \otimes u^{3} \in S^{\otimes 4}, \ldots
$$

We have that

$$
\begin{aligned}
& M_{1}=(I \otimes \underline{R} \otimes S \otimes S) \cap u_{3}^{-1}(I \otimes \underline{S} \otimes S \otimes S) \\
& M_{2}=(I \otimes \underline{R} \otimes S \otimes S) \cap u_{4}^{-1}(I \otimes \underline{S} \otimes S \otimes S) .
\end{aligned}
$$

Now take

$$
x=\sum_{i} v^{1} x_{i} \otimes v^{2} s_{i} \otimes s_{i}^{\prime} \otimes v^{3} s_{i}^{\prime \prime} \in M_{1}
$$

with $x_{i} \in I$ and $s_{i}^{\prime}, s_{i}^{\prime}, s_{i}^{\prime \prime} \in S$. From the fact that $x \in I \otimes \underline{R} \otimes S \otimes S$ and faithfully flat descent of elements (cf. [9, II.2.2]), it follows that

$$
x=\sum_{i} v^{1} x_{i} \otimes 1 \otimes v^{2} s_{i} s_{i}^{\prime} \otimes v^{3} s_{i}^{\prime \prime}
$$

and

$$
\begin{aligned}
u_{2} x & =\sum_{i} u^{1} v^{1} x_{i} \otimes 1 \otimes u^{2} v^{2} s_{i} s_{i}^{\prime} \otimes u^{3} v^{3} s_{i}^{\prime \prime} \\
& =\sum_{i} x_{i} \otimes 1 \otimes s_{i} s_{i}^{\prime} \otimes s_{i}^{\prime \prime} \in(I \otimes \underline{R} \otimes S \otimes S) .
\end{aligned}
$$

Now $u$ is a cocycle, hence $u_{2} u_{3}^{-1}=u_{4}^{-1} u_{1}$, and

$$
\begin{aligned}
u_{2} u_{3}^{-1}(I \otimes \underline{S} \otimes S \otimes S) & =u_{4}^{-1} u_{1}(I \otimes \underline{S} \otimes S \otimes S) \\
& =u_{4}^{-1}(I \otimes \underline{S} \otimes S \otimes S)
\end{aligned}
$$

and this proves that $u_{2} M_{1} \subset M_{2}$. In a similar way, we can prove that $u_{2}^{-1} M_{2} \subset M_{1}$ and $u_{2} N_{2}=N_{1}$. By extension of scalars, $\tau \otimes I_{S}: I \otimes S \rightarrow S$ is a trace. This trace restricts to a trace $M N \rightarrow S$, since $M N$ is a $T \otimes S$ subideal of $I \otimes S$, which is surjective. The composition

$$
\lambda: N \otimes M \rightarrow M N \rightarrow S
$$

is also a surjection, and we obtain a dual pair $\underline{M}=(M, N, \lambda)$ of $S$-modules, after we consider $M$ and $N$ as $S$-modules via restriction of scalars. $T$ is finitely generated as an $R$-module. Since $R$ is noetherian, the submodule $I$ of $T$ is also finitely generated, and it follows that $I \otimes R \otimes S$ is finitely 
generated as an $S$-module. $M$ and $N$ are submodules of $I \otimes R \otimes S$, and they are therefore also finitely generated as $S$-modules.

Furthermore, the map

$$
\left(u_{2}, u_{2}^{-1}\right): \underline{M}_{1} \rightarrow \underline{M}_{2}
$$

is an isomorphism of dual pairs over $S \otimes S$. To prove this, we need to check that

$$
\lambda_{1}=\lambda_{2} \circ\left(u_{2} \otimes u_{2}^{-1}\right) .
$$

Take $x \in M_{1}$ and $y \in M_{2}$, and write, with notations as above,

$$
\begin{aligned}
& x=\sum_{i} v^{1} x_{i} \otimes 1 \otimes v^{2} s_{i} s_{i}^{\prime} \otimes v^{3} s_{i}^{\prime \prime} \\
& y=\sum_{j} u^{1} y_{j} \otimes 1 \otimes u^{2} t_{j} t_{j}^{\prime} \otimes u^{3} t_{j}^{\prime \prime}
\end{aligned}
$$

with $x_{i}, y_{j} \in I$ and $s_{i}^{\prime}, s_{i}^{\prime}, s_{i}^{\prime \prime}, t_{j}^{\prime}, t_{j}^{\prime}, t_{j}^{\prime \prime} \in S$. Then

$$
\begin{aligned}
\lambda_{1}(x \otimes y) & =\left(\tau \otimes I_{S \otimes S}\right)\left(\sum_{i, j} v^{1} x_{i} u^{1} y_{j} \otimes 1 \otimes v^{2} s_{i} s_{i}^{\prime} u^{2} t_{j} t_{j}^{\prime} \otimes v^{3} s_{i}^{\prime \prime} u^{3} t_{j}^{\prime \prime}\right) \\
& =\left(\tau \otimes I_{S \otimes S}\right)\left(\sum_{i, j} x_{i} y_{j} \otimes 1 \otimes s_{i} s_{i}^{\prime} t_{j} t_{j}^{\prime} \otimes s_{i}^{\prime \prime} t_{j}^{\prime \prime}\right) \\
& =\sum_{i, j} \tau\left(x_{i} y_{j}\right) \otimes 1 \otimes s_{i} s_{i}^{\prime} t_{j} t_{j}^{\prime} \otimes s_{i}^{\prime \prime} t_{j}^{\prime \prime} \\
& =\lambda_{2}\left(\left(\sum_{i} x_{i} \otimes 1 \otimes s_{i} s_{i}^{\prime} \otimes s_{i}^{\prime \prime}\right) \otimes\left(\sum_{j} y_{j} \otimes 1 \otimes t_{j} t_{j}^{\prime} \otimes t_{j}^{\prime \prime}\right)\right) \\
& =\lambda_{2}\left(u_{2} x \otimes u_{2}^{-1} y\right) \\
& =\left(\lambda_{2} \circ\left(u_{2} \otimes u_{2}^{-1}\right)\right)(x \otimes y)
\end{aligned}
$$

$\left(u_{2}, u_{2}^{-1}\right)$ induces an isomorphism

$$
\Phi: E_{S \otimes S}\left(\underline{M}_{1}\right) \rightarrow E_{S \otimes S}\left(\underline{M}_{2}\right)
$$

and the isomorphism

$$
\Phi_{2}^{-1} \circ \Phi_{3} \circ \Phi_{1}: E_{S \otimes S \otimes S}\left(\underline{M}_{11}\right) \rightarrow E_{S \otimes S \otimes S}\left(\underline{M}_{11}\right)
$$

is induced by

$$
\begin{aligned}
\left(u_{24}^{-1} u_{25} u_{23}, u_{24} u_{25}^{-1} u_{23}^{-1}\right) & =\left(u_{32}^{-1} u_{42} u_{22}, u_{32} u_{42}^{-1} u_{22}^{-1}\right) \\
& =\left(\left(u_{2} u_{3}^{-1} u_{4}\right)_{2},\left(u_{2} u_{3}^{-1} u_{4}\right)_{2}^{-1}\right) \\
& =\left(u_{12}, u_{12}^{-1}\right) .
\end{aligned}
$$


From the fact that $\underline{M}_{11} \subset I \otimes R \otimes S^{\otimes 3}$, it follows that $\Phi_{2}^{-1} \circ \Phi_{3} \circ \Phi_{1}$ is the identity on $E_{S \otimes S \otimes S}\left(\underline{M}_{11}\right)$, and $\Phi$ is a descent datum. Thus $E_{S}(\underline{M})$ descends to a Taylor-Azumaya algebra $A$. $A$ is finitely generated as an $R$-module, because $M, N$ and $E_{S}(\underline{M})$ are finitely generated as $S$-modules.

A careful inspection of the first part of the proof of Theorem 3.3 yields that the image of $[A]$ in $H^{2}\left(R_{e t}, \mathbb{G}_{m}\right)$ is represented by $u \in Z^{2}\left(S / R, \mathbb{G}_{m}\right)$.

In the case where $R$ is not necessarily noetherian, our result follows from the fact that

$$
H^{n}\left(R_{e ́ t}, \mathbb{G}_{m}\right)=\underline{\lim } H^{n}\left(R_{0, e ́ t}, \mathbb{G}_{m}\right)
$$

where the limit runs over all noetherian subrings $R_{0}$ of $R$.

We have allready mentioned that $\operatorname{BR}(R) \subset \operatorname{Br}^{\prime}(R)$ if $R$ is noetherian. From Theorem 3.3 and Theorem 3.4, it follows immediately that this inclusion also holds for $R$ nonnoetherian.

Corollary 3.5. Let $R$ be an arbitrary commutative ring. Then $\mathrm{BR}(R) \subset$ $\operatorname{Br}^{\prime}(R)$.

Let us end with the remark that the definition of central separable algebras can be easily extended to the situation where the commutative ring $R$ is replaced by a noetherian scheme $X$ : A quasi-coherent sheaf of $\mathcal{O}_{X}$-algebras is called a central separable $\mathcal{O}_{X}$-algebra if $\Gamma(U, \mathcal{A})$ is a central separable $\Gamma\left(U, \mathcal{O}_{X}\right)$-algebra for every open affine subset $U$ of $X$. One can introduce the Brauer group $\operatorname{Br}^{\prime}(X)$ by considering equivalence classes of coherent central separable $\mathcal{O}_{X}$-algebras. Theorem 3.3 can be extended, and we obtain an embedding $\operatorname{Br}^{\prime}(X) \hookrightarrow H^{2}\left(X_{e t}, \mathbb{G}_{m}\right)$. This embedding is an isomorphism if every finite subset of $X$ is contained in an open affine subset of $X$ (in this situation, Artin's Refinement Theorem holds, and this is sufficient to generalize the proof of Theorem 3.4).

\section{References}

[1] M. Artin, On the joins of Hensel rings, Adv. in Math., 7 (1971), 282-296.

[2] M. Auslander and O. Goldman, The Brauer group of a commutative ring, Trans. Amer. Math. Soc., 97 (1960), 367-409.

[3] S. Caenepeel, Brauer groups, Hopf algebras, and Galois theory, K-monographs in Math., 4, Kluwer Academic Publishers, Dordrecht, 1998.

[4] F. DeMeyer and E. Ingraham, Separable algebras over commutative rings, Lecture Notes in Math., 181, Springer Verlag, Berlin, 1971.

[5] O. Gabber, Some Theorems on Azumaya algebras, in 'Groupe de Brauer', M. Kervaire and M. Ojanguren (Eds.), Lecture Notes in Math., 844, Springer Verlag, Berlin, 1981. 
[6] F. Grandjean, Les algèbres d'Azumaya sans unité, Thesis, Université Catholique de Louvain (UCL), Louvain-la-Neuve, 1997.

[7] A. Grothendieck, Le groupe de Brauer I, II, III in 'Dix Exposés sur la cohomologie des schémas', North Holland, Amsterdam, 1968.

[8] R. Hoobler, When is $\operatorname{Br}(X)=\operatorname{Br}^{\prime}(X)$ ?, in 'Brauer groups in ring theory and algebraic geometry', F. Van Oystaeyen and A. Verschoren (Eds.), Lecture Notes in Math., 917, Springer Verlag, Berlin, (1982), 231-245.

[9] M.A. Knus and M. Ojanguren, Théorie de la descente et algèbres d'Azumaya, Lecture Notes in Math., 389, Springer Verlag, Berlin, 1974.

[10] J.S. Milne, Etale cohomology, Princeton University Press, Princeton, 1980.

[11] M. Orzech and C. Small, The Brauer group of a commutative ring, Marcel Dekker, Inc., New York, 1975.

[12] I. Raeburn and J.L. Taylor, The bigger Brauer group and étale cohomology, Pacific J. Math., 119 (1985), 445-463.

[13] M. Raynaud, Anneaux locaux Henséliens, Lecture Notes in Math., 169, Springer Verlag, Berlin, 1971.

[14] J.L. Taylor, A bigger Brauer group, Pacific J. Math., 103 (1982), 163-203.

[15] E.M. Vitale, The Brauer and Brauer-Taylor groups of a symmetric monoidal category, Cahiers de Topologie et Géometrie Différentielle Catégoriques, 37 (1996), 91-122.

Received March 5, 1997 and revised August 1, 1997.

Free University of Brussels, VUB

B-1050 Brussels, Belgium

E-mail address: scaenepe@vub.ac.be

AND

Université Catholique de Louvain, UCL

B-1348 Louvain-LA-Neuve, Belgium

E-mail address: Grandjean@agel.ucl.ac.be 